\title{
Perfil epidemiológico dos indivíduos com tuberculose em um centro de referência terciário e fatores associados aos resultados da baciloscopia e da radiografia do tórax
}

\author{
Epidemiological profile of individuals with tuberculosis in a tertiary referral center and \\ factors associated with the results of sputum smear microscopy and chest radiography \\ Perfil epidemiológico de individuos con tuberculosis en un centro de referencia terciario y \\ factores asociados con los resultados de la microscopía de esputo y la radiografía de \\ tórax
}

Wesley Murilo de Abreu Roveda ${ }^{1}$, Marina Almeida de Mendonça ${ }^{1}$, Jenaine Rosa Godinho ${ }^{2}$, Camila de Paula Ceccatto ${ }^{2}$, Geisa Fregona ${ }^{2}$, Veronica Lourenço Wittmer ${ }^{1}$, Halina Duarte ${ }^{1}$, Marcela Cangussu Barbalho-Moulim¹, Flávia Marini Paro ${ }^{1 *}$.

\section{RESUMO}

Objetivo: Descrever as características sociodemográficas e clínicas dos indivíduos com tuberculose (TB), e avaliar associações dessas características com os resultados da baciloscopia e radiografia de tórax. Métodos: Estudo descritivo transversal, com amostra constituída pelos indivíduos diagnosticados com TB e notificados em um centro de referência terciário estadual brasileiro. Dados sociodemográficos e clínicos foram coletados dos prontuários físicos, eletrônicos e do SINAN. Para a análise estatística foram utilizados o Quiquadrado, o teste Exato de Fisher e a Razão da Máxima Verossimilhança, sendo considerado significante $p<0,05$. Resultados: Houve maior prevalência de indivíduos do sexo masculino, adultos, da região Metropolitana, com TB pulmonar. Comorbidades encontradas: tabagismo $(42,86 \%)$, etilismo $(40,81 \%)$, hipertensão arterial sistêmica (28,6\%), doença hepática (26,53\%), diabetes mellitus $(24,5 \%)$, HIV $(12,2 \%)$ e drogas ilícitas $(10,20 \%)$. A baciloscopia positiva foi associada com maior percentual de cultura positiva e de TB multirresistente (MDR), sendo encontrada em todos os indivíduos TB MDR. A radiografia de tórax suspeita foi observada em todos os indivíduos TB pulmonar. Conclusão: As comorbidades mais frequentes foram: tabagismo, hipertensão arterial sistêmica, doença hepática e diabetes mellitus. A associação da baciloscopia positiva com TB MDR merece atenção, considerando que os indivíduos bacilíferos possuem maior potencial de transmissibilidade da doença.

Palavras-chave: Epidemiologia, Pneumopatias, Tuberculose.

\section{ABSTRACT}

Objective: To describe the sociodemographic and clinical characteristics of individuals with tuberculosis (TB), and to evaluate associations of these characteristics with the results of sputum smear microscopy and chest radiography. Methods: This is a descriptive cross-sectional study, with a sample consisting of individuals diagnosed with TB and notified at a tertiary referral center from a Brazilian state. Sociodemographic and clinical data were collected from physical and electronic medical records and SINAN. Chi-square, Fisher's Exact test, and Maximum Likelihood Ratio were used for statistical analysis. Significance was set at $p<0.05$. Results: There was a higher prevalence of males, adults, from the Metropolitan region, with pulmonary TB. Comorbidities found: smoking (42.86\%), alcoholism (40.81\%), systemic arterial hypertension (28.6\%), liver disease (26.53\%), diabetes mellitus (24.5\%), HIV (12.2\%) and illicit drugs use (10.20\%). Positive sputum smear microscopy was associated with a higher percentage of positive culture and multidrug-resistant TB (MDR), being found in all MDR TB individuals. Suspected chest radiography was seen in all pulmonary TB individuals. Conclusion: The most frequent comorbidities were: smoking, systemic arterial hypertension, liver disease and diabetes. The association of positive smear microscopy with MDR TB deserves attention, considering that bacilliferous individuals have a greater potential for disease transmissibility.

Key words: Epidemiology, Lung diseases, Tuberculosis.

1Universidade Federal do Espírito Santo (UFES), Vitória - ES. *E-mail: flamarp@yahoo.com

2 Hospital Universitário Cassiano Antonio de Moraes (HUCAM), Vitória - ES.

SUBMETIDO EM: 4/2020

PUBLICADO EM: 7/2020 


\section{RESUMEN}

Objetivo: Describir las características sociodemográficas y clínicas de las personas con tuberculosis (TB) y evaluar las asociaciones de estas características con los resultados de la microscopía de esputo y la radiografía de tórax. Métodos: Estudio descriptivo transversal, con muestra compuesta por individuos con TB notificados en un centro de referencia terciario estatal brasileño. Se recogieron datos de registros físicos, electrónicos y SINAN. Para el análisis estadístico, se utilizaron Chi-cuadrado, prueba exacta de Fisher y cociente de probabilidad máxima ( $p<0,05$ considerado significativo). Resultados: Mayor prevalencia de varones, adultos, de la región metropolitana, con TB pulmonar. Comorbilidades encontradas: tabaquismo $(42,86 \%)$, alcoholismo $(40,81 \%)$, hipertensión arterial sistémica $(28,6 \%)$, enfermedad hepática $(26,53 \%)$, diabetes mellitus (24,5\%), $\mathrm{VIH}(12,2 \%)$ y drogas ilícitas (10,20\%). La microscopía de esputo positiva se asoció con un mayor porcentaje de cultivo positivo y TB multirresistente (MDR), que se encuentra en todos los individuos con TB MDR. Se sospechó radiografía de tórax en todos los individuos con tuberculosis pulmonar. Conclusión: Las comorbilidades más frecuentes fueron: tabaquismo, hipertensión arterial, enfermedad hepática y diabetes. La asociación de la microscopía de frotis positiva con TB MDR merece atención, considerando que las personas bacilíferas tienen un mayor potencial de transmisión de la enfermedad.

Palabras clave: Epidemiología, Enfermedades pulmonares, Tuberculosis.

\section{INTRODUÇÃO}

A tuberculose (TB) constitui um importante problema de saúde pública mundial, sendo uma das infecções crônicas com maior índice de morbidade e mortalidade. Embora existam registros da doença em todos os países, aproximadamente $87 \%$ dos casos estão concentrados em 30 países, entre os quais, o Brasil ocupa a 20ª posição (WORLD HEALTH ORGANIZATION, 2019).

Em 2018, foram diagnosticados 72.788 casos novos de TB no Brasil, o que corresponde a um coeficiente de incidência de 34,8 casos/100 mil habitantes. No estado do Espírito Santo (ES), a prevalência em 2018, foi de 1.218 casos de TB, perfazendo um coeficiente de incidência de 30 casos/100 mil habitantes, sendo a maioria dos casos na Região Metropolitana (, onde se localiza o único Centro de Referência Terciário estadual para o tratamento de TB (CRT-TB) e a capital do estado (BRASIL, 2019; FREGONA G, et al, 2017). No Brasil, a rede de atenção à saúde é regionalizada e hierarquizada em níveis de complexidade crescente, privilegiando, a descentralização para a atenção básica, o que amplia o acesso da população geral e das populações sob maior risco de contrair TB. Casos com evolução clínica desfavorável, resistência aos fármacos antiTB e micobactérias não tuberculosas são encaminhados para um CRT-TB (BRASIL, 2018).

$O$ enfrentamento da TB exige abordagem global, regional, transfronteiriça e específica, com o reconhecimento das diferenças existentes entre e dentro dos países, como endemicidade, tendências sociodemográficas, urbanização, características individuais e os desafios para identificar e tratar todos os indivíduos com TB em cada região (WORLD HEALTH ORGANIZATION, 2017). Portanto, são fundamentais estudos que avaliem as características demográficas e clínicas dos indivíduos com TB, nas diferentes regiões e CRT-TB, para que se possa planejar e implementar estratégias resolutivas e efetivas para as especificidades de cada população.

O principal objetivo deste estudo foi descrever o perfil sociodemográfico e clínico dos indivíduos com diagnóstico de TB, atendidos em um CRT-TB. Foi objetivo secundário avaliar a associação das características sociodemográficas e clínicas com os resultados da baciloscopia e da radiografia de tórax.

\section{MÉTODOS}

Trata-se de um estudo descritivo transversal, cujos dados foram coletados nos prontuários dos indivíduos diagnosticados com TB, que ingressaram no ambulatório de pneumologia de um hospital universitário, onde está localizado o único CRT-TB do estado do ES. Foram incluídos no estudo todos os indivíduos admitidos no ambulatório, no período de fevereiro a julho de 2019, com suspeita de TB. Foram excluídos indivíduos que não tiveram o diagnóstico de TB confirmado e notificado.

O estudo foi aprovado pelo Comitê de Ética em Pesquisa do HUCAM (CAAE 04163318.8.0000.571, parecer 04163318). Todos os procedimentos foram conduzidos de acordo a Declaração de Helsinque, suas 
atualizações, e com a Resolução no 466/12, que regulamenta as pesquisas envolvendo seres humanos no Brasil.

Foram coletados, dos prontuários físicos, eletrônicos e SINAN, os dados sociodemográficos (idade, sexo, escolaridade, raça, estado civil, residência, regionalização estadual e municipal de saúde) e as variáveis clínicas: diagnóstico, resultados da baciloscopia, cultura, radiografia de tórax, esquema de tratamento, infecção por HIV, hipertensão arterial sistêmica (HAS), diabetes mellitus (DM), doença hepática, alcoolismo, tabagismo e uso de drogas ilícitas.

$\mathrm{Na}$ análise descritiva, variáveis categóricas foram expressas pelas suas frequências absolutas e relativas e variáveis quantitativas foram apresentadas como média e desvio padrão. A comparação entre duas variáveis categóricas foi feita com o teste Qui-quadrado, exceto se resultados menores do que cinco fossem esperados, situações em que foi usado o teste Exato de Fisher. A Razão da Máxima Verossimilhança foi usada para variáveis com mais de duas categorias. Foi considerado significante $p<0,05$.

\section{RESULTADOS}

No período de 01 de fevereiro a 31 de julho de 2019, 56 indivíduos foram admitidos no CRT-TB. Após os procedimentos diagnósticos, sete indivíduos foram diagnosticados com micobactérias não tuberculosas, sendo excluídos do estudo. A amostra foi constituída pelos 49 indivíduos, com diagnóstico de TB confirmado e notificado. Os dados sociodemográficos mostraram maior prevalência de indivíduos do sexo masculino $(55,1 \%)$, pardos $(77,6 \%)$, casados $(44,9 \%)$, com ensino médio completo $(30,6 \%)$ e residentes na macrorregião Metropolitana $(71,4 \%)$, na qual fica localizado o CRT-TB. A média de idade encontrada foi de $47,69( \pm 18,13)$ anos, variando de 16 a 84 anos. O mês predominante de admissão de indivíduos com TB foi fevereiro $(24,5 \%)$ (Tabela 1).

Com relação às características clínicas da amostra, houve predomínio de indivíduos com a forma pulmonar (51\%). Nos 20 indivíduos que apresentavam apenas a forma extrapulmonar, foi observada a seguinte distribuição: ganglionar periférica $(n=9 ; 37,5 \%)$, ocular $(n=4 ; 16,7 \%)$, óssea $(n=1 ; 4,2 \%)$, pericárdica $(n=2$; $8,3 \%)$, pleural $(n=4 ; 16,7 \%)$, pleural e pericárdica $(n=1 ; 4,2 \%)$, e outras não especificadas $(n=3 ; 12,5 \%)$. Nos quatro indivíduos que apresentavam a forma pulmonar concomitante à extrapulmonar foi encontrada a seguinte distribuição: ganglionar periférica $(n=2 ; 50 \%)$, pleural $(n=1 ; 25 \%)$ e outra não especificada $(n=1$; $25 \%$ ). A prevalência de Tuberculose Multirresistente (TB-MDR) foi de 10,2\%. A prevalência de coinfecção TBHIV foi de $12,2 \%$. Foram observadas as seguintes prevalências de outras comorbidades: HAS (28,6\%), doença hepática $(26,53 \%)$, tendo como principal causa a hepatotoxidade ao esquema de tratamento $(69,2 \%)$, e DM (24,5\%). Além disso, $42,86 \%$ dos indivíduos eram tabagistas ou ex-tabagistas; $40,81 \%$ eram etilistas ou ex-etilistas; e 10,20\% eram usuários de drogas ilícitas (Tabela 2).

A Tabela 3 mostra os resultados dos testes estatísticos que avaliaram a associação da baciloscopia com outras variáveis. No grupo de indivíduos com baciloscopia positiva, houve um maior percentual de cultura positiva $(71,4 \%)$ do que no grupo com baciloscopia negativa $(35,0 \%), p=0,19$. Todos os indivíduos TB-MDR apresentaram baciloscopia positiva. Além disso, no cruzamento da baciloscopia com a classificação da TB, foi observada maior proporção de baciloscopia positiva no grupo de indivíduos com TB-MDR, do que no grupo com TB sem resistência aos medicamentos, $p=0,049$. Não houve associação da baciloscopia com as variáveis escolaridade $(p=0,319)$ e macrorregião de saúde $(p=0,2)$, não apresentadas na tabela. Também não foram encontradas associações da baciloscopia com as demais variáveis que constam na tabela.

A Tabela 4 mostra os resultados dos testes que avaliaram a associação da radiografia de tórax com as demais variáveis. Todos os indivíduos com diagnóstico de TB pulmonar, associada ou não à extrapulmonar, apresentaram diagnóstico suspeito na radiografia. No grupo de indivíduos com as formas pulmonar e pulmonar/extrapulmonar houve maior percentual de radiografias suspeitas $(100 \%)$ do que no grupo de indivíduos com a forma extrapulmonar $(41,2 \%), p<0,01$. Além disso, no grupo de indivíduos com tipo pleural e pleural/pericárdica houve maior percentual de radiografias suspeitas $(100 \%)$ do que nos grupos: ganglionar periférica $(44,4 \%)$ e outras $(37,5 \%), p=0,047$. Não foram observadas associações da radiografia com as outras variáveis. 
Tabela 1 - Características sociodemográficas dos indivíduos com tuberculose atendidos em um centro de referência estadual terciário, $n=49$. Vitória - ES.

\begin{tabular}{lc}
\hline Variável & $\mathbf{N}(\%)$ \\
\hline Sexo & $27(55,1)$ \\
\hline Masculino & $22(44,9)$ \\
Feminino & \\
\hline Idade & $21(42,9)$ \\
\hline$<40$ & $17(34,7)$ \\
40 a 64 & $11(22,4)$ \\
$\geq 65$ & \\
\hline Raça & $9(18,4)$ \\
\hline Branca & $1(2,0)$ \\
Negra & $38(77,6)$ \\
Parda & $1(2,0)$ \\
Sem informação &
\end{tabular}

\begin{tabular}{lc}
\hline Estado Civil & \\
\hline Solteiro & $21(42,9)$ \\
Casado & $22(44,9)$ \\
Divorciado/Viúvo & $6(12,2)$ \\
\hline
\end{tabular}

\begin{tabular}{lc}
\hline Escolaridade & \\
\hline Nenhuma/ Ensino fundamental incompleto & $11(22,5)$ \\
Ensino fundamental completo & $3(6,1)$ \\
Ensino médio completo & $15(30,6)$ \\
Ensino superior completo & $5(10,2)$ \\
Sem informação & $15(30,6)$ \\
\hline Mês de Notificação & \\
\hline Fevereiro & $12(24,5)$ \\
Março & $9(18,4)$ \\
Abril & $4(8,2)$ \\
Maio & $8(16,3)$ \\
Junho & $8(16,3)$ \\
Julho & $8(16,3)$ \\
\hline Regiões de Saúde do ES & $2(4,1)$ \\
\hline Norte & $5(10,2)$ \\
Central & $35(71,4)$ \\
Metropolitana & $7(14,3)$ \\
Sul &
\end{tabular}

Fonte: Roveda WMA, et al., 2019. 
Tabela 2 - Características clínicas de indivíduos com tuberculose atendidos em um centro de referência estadual terciário do Brasil, n=49. Vitória - ES, 2019.

\begin{tabular}{|c|c|}
\hline Variável & $\mathbf{N}(\%)$ \\
\hline \multicolumn{2}{|l|}{ Forma Clínica } \\
\hline Pulmonar & $25(51,0)$ \\
\hline Extrapulmonar & $20(40,8)$ \\
\hline Pulmonar / Extrapulmonar & $4(8,2)$ \\
\hline \multicolumn{2}{|l|}{ Classificação } \\
\hline Tuberculose & $44(89,8)$ \\
\hline Tuberculose multirresistente & $5(10,2)$ \\
\hline \multicolumn{2}{|l|}{ Baciloscopia } \\
\hline Positiva & $23(46,9)$ \\
\hline Negativa & $23(46,9)$ \\
\hline Não realizada & $3(6,2)$ \\
\hline \multicolumn{2}{|l|}{ Cultura } \\
\hline Positiva & $22(44,9)$ \\
\hline Negativa & $19(38,8)$ \\
\hline Sem informação & $8(16,3)$ \\
\hline \multicolumn{2}{|l|}{ Radiografia de Tórax } \\
\hline Suspeito & $35(71,4)$ \\
\hline Normal & $10(20,4)$ \\
\hline Outra patologia & $2(4,1)$ \\
\hline Não realizada & $2(4,1)$ \\
\hline \multicolumn{2}{|l|}{ HIV } \\
\hline Negativo & $43(87,8)$ \\
\hline Positivo & $6(12,2)$ \\
\hline \multicolumn{2}{|l|}{ Diabetes Mellitus } \\
\hline Não & $34(69,4)$ \\
\hline Sim & $12(24,5)$ \\
\hline Sem informação & $3(6,1)$ \\
\hline \multicolumn{2}{|c|}{ Hipertensão arterial sistêmica } \\
\hline Não & $31(63,3)$ \\
\hline $\operatorname{Sim}$ & $14(28,6)$ \\
\hline Sem informação & $4(8,1)$ \\
\hline \multicolumn{2}{|l|}{ Doença Hepática } \\
\hline Não & $35(71,4)$ \\
\hline Sim & $13(26,5)$ \\
\hline Sem informação & $1(2,1)$ \\
\hline \multicolumn{2}{|l|}{ Tabagista } \\
\hline Não & $26(53,1)$ \\
\hline Sim & $8(16,3)$ \\
\hline Ex-tabagista & $13(26,5)$ \\
\hline Sem informação & $2(4,1)$ \\
\hline \multicolumn{2}{|l|}{ Etilista } \\
\hline Nega & $27(55,1)$ \\
\hline Sim & $14(28,6)$ \\
\hline Ex-etilista & $6(12,2)$ \\
\hline Sem informação & $2(4,1)$ \\
\hline \multicolumn{2}{|l|}{ Drogas Ilícitas } \\
\hline Nega & $44(89,8)$ \\
\hline Sim & $5(10,2)$ \\
\hline
\end{tabular}

Fonte: Roveda WMA, et al., 2019. 
Tabela 3 - Associação das variáveis demográficas e clínicas com os resultados da baciloscopia.

\begin{tabular}{|c|c|c|c|}
\hline \multirow{2}{*}{ Variável } & \multicolumn{2}{|c|}{ Baciloscopia } & \multirow{2}{*}{ p-valor } \\
\hline & Positiva n (\%) & Negativa n (\%) & \\
\hline \multicolumn{4}{|l|}{ Sexo } \\
\hline Masculino & $15(65,2)$ & $11(47,8)$ & \multirow{2}{*}{$0,234^{*}$} \\
\hline Feminino & $8(34,8)$ & $12(52,2)$ & \\
\hline \multicolumn{4}{|l|}{ Faixa etária } \\
\hline$<39$ & $10(43,5)$ & $9(39,1)$ & \multirow{3}{*}{$0,392^{*}$} \\
\hline 40 a 64 & $6(26,1)$ & $10(43,5)$ & \\
\hline$>64$ & $7(30,4)$ & $4(17,4)$ & \\
\hline \multicolumn{4}{|l|}{ Raça / Cor } \\
\hline Branca & $5(22,7)$ & $4(17,4)$ & \multirow{2}{*}{$0,722^{* *}$} \\
\hline Negra / Parda & $17(77,3)$ & $19(82,6)$ & \\
\hline \multicolumn{4}{|l|}{ Forma Clínica } \\
\hline Pulmonar e Pulmonar / Extrapulmonar & $17(73,9)$ & $11(47,8)$ & \multirow{2}{*}{$0,070^{*}$} \\
\hline Extrapulmonar & $6(26,1)$ & $12(52,2)$ & \\
\hline \multicolumn{4}{|l|}{ Tipo de Extrapulmonar } \\
\hline Ganglionar periférica & $3(50,0)$ & $6(40,0)$ & \multirow{3}{*}{$0,128^{* * *}$} \\
\hline Pleural / Pleural e Pericárdica & - & $5(33,3)$ & \\
\hline Outras & $3(50,0)$ & $4(26,7)$ & \\
\hline \multicolumn{4}{|l|}{ Classificação } \\
\hline Tuberculose & $18(78,3)$ & $23(100,0)$ & \multirow{2}{*}{$0,049^{* *}$} \\
\hline Tuberculose multirresistente & $5(21,7)$ & - & \\
\hline \multicolumn{4}{|l|}{ Cultura } \\
\hline Positiva & $15(71,4)$ & $7(35,0)$ & \multirow{2}{*}{$0,019^{*}$} \\
\hline Negativa & $6(28,6)$ & $13(65,0)$ & \\
\hline \multicolumn{4}{|l|}{ HIV } \\
\hline Negativo & $20(87,0)$ & $20(87,0)$ & \multirow{2}{*}{$1,000^{* *}$} \\
\hline Positivo & $3(13,0)$ & $3(13,0)$ & \\
\hline \multicolumn{4}{|l|}{ Diabetes Mellitus } \\
\hline Não & $16(76,2)$ & $16(72,7)$ & \multirow{2}{*}{$0,795^{\star}$} \\
\hline $\operatorname{Sim}$ & $5(23,8)$ & $6(27,3)$ & \\
\hline \multicolumn{4}{|l|}{ Tabagismo } \\
\hline Nega & $10(45,5)$ & $14(60,9)$ & \multirow{3}{*}{$0,510^{* * *}$} \\
\hline Sim & $4(18,2)$ & $4(17,4)$ & \\
\hline Ex-Tabagista & $8(36,4)$ & $5(21,7)$ & \\
\hline \multicolumn{4}{|l|}{ Etilismo } \\
\hline Nega & $10(45,5)$ & $15(65,2)$ & \multirow{3}{*}{$0,342^{* * *}$} \\
\hline Sim & $9(40,9)$ & $5(21,7)$ & \\
\hline Ex-Etilista & $3(13,6)$ & $3(13,0)$ & \\
\hline Uso de Drogas Ilícitas & & & \\
\hline Não & $20(87,0)$ & $22(95,7)$ & \\
\hline Sim & $3(13,0)$ & $1(4,3)$ & $0,608^{\star \star}$ \\
\hline Radiografia de Tórax & & & \\
\hline Suspeito & $18(85,7)$ & $16(76,2)$ & \\
\hline Normal & $3(14,3)$ & $5(23,8)$ & $0,697^{\approx \pi}$ \\
\hline
\end{tabular}

*Teste Qui-quadradro;

**Teste Exato de Fisher;

***Razão de Verossimilhança.

Fonte: Roveda WMA, et al., 2019. 
Tabela 4 - Associação das variáveis demográficas e clínicas com o resultado da radiografia de tórax.

\begin{tabular}{|c|c|c|c|}
\hline \multirow{2}{*}{ Variável } & \multicolumn{2}{|c|}{ Radiografia do Tórax } & \multirow{2}{*}{ p-valor } \\
\hline & Suspeito n (\%) & Normal n (\%) & \\
\hline \multicolumn{4}{|l|}{ Sexo } \\
\hline Masculino & $19(54,3)$ & $6(60,0)$ & \multirow{2}{*}{$1,000^{* *}$} \\
\hline Feminino & $16(45,7)$ & $4(40,0)$ & \\
\hline \multicolumn{4}{|l|}{ Faixa etária } \\
\hline$<39$ & $14(40,0)$ & $6(60,0)$ & \multirow{3}{*}{$0,468^{* * *}$} \\
\hline 40 a 64 & $13(37,1)$ & $3(30,0)$ & \\
\hline$>64$ & $8(22,9)$ & $1(10,0)$ & \\
\hline \multicolumn{4}{|l|}{ Raça / Cor } \\
\hline Branca & $7(20,6)$ & $2(20,0)$ & \multirow{2}{*}{$1,000^{* *}$} \\
\hline Negra / Parda & $27(79,4)$ & $8(80,0)$ & \\
\hline \multicolumn{4}{|l|}{ Forma Clínica } \\
\hline Pulmonar e Pulmonar / Extrapulmonar & $28(80,0)$ & - & \multirow{2}{*}{$0,000^{* *}$} \\
\hline Extrapulmonar & $7(20,0)$ & $10(100,0)$ & \\
\hline \multicolumn{4}{|l|}{ Tipo de Extrapulmonar } \\
\hline Ganglionar periférica & $4(36,4)$ & $5(50,0)$ & \multirow{3}{*}{$0,047^{* * *}$} \\
\hline Pleural / Pleural e Pericárdica & $4(36,4)$ & - & \\
\hline Outras & $3(27,3)$ & $5(50,0)$ & \\
\hline \multicolumn{4}{|l|}{ Classificação } \\
\hline $\mathrm{TB}$ & $30(85,7)$ & $10(100,0)$ & \multirow{2}{*}{$0,571^{* *}$} \\
\hline TBMR & $5(14,3)$ & - & \\
\hline \multicolumn{4}{|l|}{ Cultura } \\
\hline Positiva & $19(61,3)$ & $1(16,7)$ & \multirow{2}{*}{$0,075^{\star \star}$} \\
\hline Negativa & $12(38,7)$ & $5(83,3)$ & \\
\hline \multicolumn{4}{|l|}{ Diabetes Mellitus } \\
\hline Não & $22(68,8)$ & $8(80,0)$ & \multirow{2}{*}{$0,696^{\star *}$} \\
\hline Sim & $10(31,2)$ & $2(20,0)$ & \\
\hline \multicolumn{4}{|l|}{ Tabagismo } \\
\hline Nega & $18(52,9)$ & $5(55,6)$ & \multirow{3}{*}{$0,774^{\star * *}$} \\
\hline Sim & $7(20,6)$ & $1(11,1)$ & \\
\hline Ex-Tabagista & $9(26,5)$ & $3(33,3)$ & \\
\hline \multicolumn{4}{|l|}{ Etilismo } \\
\hline Nega & $19(55,9)$ & $5(55,6)$ & \multirow{3}{*}{$0,685^{\star * *}$} \\
\hline Sim & $11(32,4)$ & $2(22,2)$ & \\
\hline Ex-Etilista & $4(11,8)$ & $2(22,2)$ & \\
\hline \multicolumn{4}{|l|}{ Uso de Drogas llícitas } \\
\hline Não & $32(91,4)$ & $8(80,0)$ & \multirow{2}{*}{$0,306^{\star *}$} \\
\hline Sim & $3(8,6)$ & $2(20,0)$ & \\
\hline
\end{tabular}

* Teste Qui-quadradro;

** Teste Exato de Fisher;

*** Razão de Máxima Verossimilhança.

Fonte: Roveda WMA, et al., 2019. 


\section{DISCUSSÃO}

Os resultados mostraram maior prevalência de TB em homens, adultos, o que também tem sido observado em outros estudos nacionais e internacionais (WORLD HEALTH ORGANIZATION, 2019; BRASIL, 2019; FERNANDES FMC, et al., 2017; OLIVA HNP, 2018). Além disso, a maioria dos indivíduos era da raça parda e o grau de escolaridade predominante foi ensino médio completo. Resultados semelhantes foram relatados no Distrito Federal do Brasil, onde também houve maior prevalência de TB em homens, adultos e pardos, contudo, predominou escolaridade primária (40,2\%) (FERNANDES FMC, et al., 2017).

Muitos fatores aumentam a suscetibilidade à infecção por Mycobacterium tuberculosis, como HIV, DM, tabagismo, alcoolismo e o uso de drogas ilícitas. Fatores, cuja prevalência deve ser conhecida em cada população, para que a estruturação dos serviços de saúde contemple as especificidades regionais (SILVA DR, et al., 2018;). Além do HIV/AIDS, outras condições de saúde que comprometam o sistema imunológico, também contribuem para o risco aumentado de TB, com maior gravidade e pior prognóstico (CRABTREERAMÍREZ B, et al, 2019; KUMARI P e MEENA LS, 2014; LEONG LY, et al., 2019).

A TB é a principal causa de morte nos indivíduos com HIV/AIDS (WORLD HEALTH ORGANIZATION, 2017), pois a imunossupressão aumenta a chance de infecção, e predispõe a desfechos desfavoráveis (BRASIL, 2019), razão pela qual, a coinfecção TB-HIV deve ser avaliada em todos os indivíduos. Segundo a OMS, em 2018, 8,6\% dos indivíduos com TB apresentavam HIV (WORLD HEALTH ORGANIZATION, 2019). No Brasil, em 2016, 83,1 \% dos indivíduos com TB foram testados para o HIV, entre os quais, $12,9 \%$ tinham a coinfecção TB-HIV. No presente estudo, a prevalência de TB-HIV foi de $12,2 \%$, semelhante aos dados nacionais (BRASIL, 2016).

Indivíduos com TB têm risco aumentado de desenvolver síndrome coronariana aguda (SCA) (CHUNG W$S$, et al., 2014). Um modelo mecanicista potencial para explicar essa associação é baseado em pesquisas que mostram ativação imune persistente na TB latente e na TB ativa, com a possibilidade de reação cruzada dos anticorpos com os autoantígenos presentes nos vasos sanguíneos humanos, levando à autoimunidade. A inflamação relacionada à infecção pode ter impacto em diferentes etapas da doença coronariana, desde a formação inicial da placa até sua ruptura, mas estudos são necessários para elucidar melhor esses mecanismos fisiopatológicos (HUAMAN MA, et al., 2015). Os profissionais de saúde devem estar cientes dessas evidências, para que possam atuar no sentido de reduzir os fatores de risco modificáveis para SCA nos pacientes com TB (CHUNG W-S, et al., 2014).

HAS, dislipidemia, obesidade, sedentarismo, tabagismo e DM são considerados alguns dos principais fatores de risco para doenças coronarianas (PRÉCOMA DB, et., 2019). Para que as estratégias de prevenção e redução dos fatores de risco cardiovasculares em pacientes com TB possam ser planejadas de forma efetiva, é necessário que se conheça a sua prevalência na população com TB atendida nos diferentes serviços e regiões. Entre os fatores de risco modificáveis mencionados, HAS, DM e tabagismo tiveram sua prevalência descrita no presente estudo e serão discutidos nos próximos parágrafos.

A HAS apresentou prevalência de $28,6 \%$ nos pacientes do CRT-TB, mas a prevalência de HAS nos indivíduos com TB varia nas diferentes regiões (CHUNG W-S, et al., 2014; SEEGERT AB, et al, 2017). Uma revisão sistemática que objetivou avaliar a associação entre TB e HAS, não encontrou evidência para apoiar essa relação, e sugeriu que mais estudos são necessários para avaliar se há risco aumentado de HAS entre indivíduos com TB, e também se a HAS aumenta o risco de desenvolver a infecção por TB (SEEGERT AB, et al, 2017). Apesar dessa relação não ser conhecida, o diagnóstico, tratamento e controle da HAS nos indivíduos com TB é fundamental, considerando que a HAS é um dos mais importantes fatores de risco para SCA.

A DM, além de constituir um fator de risco para SCA, aumenta o risco de os indivíduos desenvolverem TB ativa (JEON CY e MURRAY MB, 2008; LEUNG CC, et al., 2008). Além disso, piora seu prognóstico, especialmente em países endêmicos para TB e com taxas crescentes de prevalência de DM (UGARTE-GIL C, et al., 2020), como é o caso do Brasil. Um estudo recente, realizado em quatro países, apresentou prevalência de 12,5\% de DM entre os indivíduos com TB (UGARTE-GIL C, et al., 2020). Na nossa amostra, essa prevalência foi de $24,5 \%$, maior do que a observada em outros estudos nacionais e internacionais 
(UGARTE-GIL C, et al., 2020; ROCHA et al., 2016). Uma provável explicação para essa maior prevalência, é que a associação entre DM e TB leva a complicações múltiplas, que podem aumentar a necessidade do tratamento em um CRT-TB (BRASIL, 2018), sendo, portanto esperada maior prevalência de DM na população com TB atendida nesses locais, como é o caso do presente estudo. A prevalência de DM e as características clínicas dos indivíduos com TB e DM também diferem consideravelmente entre os países (UGARTE-GIL C, et al., 2020), devendo ser estudadas em cada região e serviço, para que seja possível um controle mais efetivo das duas doenças.

Foi observada alta prevalência de doença hepática, o que é justificável, visto que é a principal causa foi a hepatotoxicidade por medicamento antiTB $(69,22 \%)$, uma complicação do tratamento descrita na literatura, que constitui um importante motivo para referenciamento ao CRT-TB (BRASIL, 2018). Além disso, faz-se necessário ressaltar que $53,8 \%$ dos indivíduos que apresentavam doença hepática eram etilistas ou exetilistas, o que pode ter contribuído para esta complicação, considerando que o etilismo é uma das principais causas conhecidas de cirrose hepática (HADDAD L, et al., 2018).

Para o planejamento das estratégias de controle da TB, merece atenção o fato de que, no presente estudo, $40,8 \%$ dos indivíduos com TB eram etilistas $(28,6 \%)$ ou ex-etilistas $(12,2 \%)$, pois uma recente revisão sistemática com metanálise, que avaliou a associação do álcool com o aumento do risco de TB, concluiu que o uso do álcool e os problemas relacionados ao seu consumo foram associados a um risco aumentado de TB, sugerindo que as políticas de saúde relacionadas ao controle do etilismo devem desempenhar um papel fundamental nas estratégias de controle da TB (IMTIAZ, et al., 2017) .

O fumo é um dos principais fatores associados à ocorrência de TB (KHAN AH, et al., 2015). Em um estudo realizado nos Estados Unidos, a prevalência de tabagismo ou ex-tabagismo, nos indivíduos com TB, foi de 28\% (CARAFFA E, et al., 2016). Segundo dados do DATASUS, no ES houve prevalência de $31 \%$ de casos de TB associada ao tabagismo, em 2018 (BRASIL, MS/SVS, 2018). A alta prevalência de indivíduos tabagistas observada no presente estudo $(42,8 \%)$, em comparação com os dados nacionais, pode ser decorrente do aumento da necessidade de tratamento em um CRT-TB nesses indivíduos, devido às complicações associadas ao tabagismo que estão descritas na literatura (SILVA DR, et al., 2018; KHAN AH, et al., 2015; ARNEDO-PENA A, et al., 2019).

A utilização de drogas como o crack/cocaína, devido às suas condições de uso, favorece a disseminação da TB (CRUZ VD, et al., 2013). Em nossa amostra, houve prevalência de 10,2\% de usuários de drogas ilícitas. Um estudo realizado no mesmo CRT-TB, no período de 2002 a 2012, apresentou prevalência de 13\% (FREGONA G, et al., 2017), demonstrando que mesmo com o passar de quase duas décadas, o perfil de indivíduos ingressantes no centro de referência estudado, apresentou pequena alteração no percentual de uso de drogas ilícitas, embora essa prevalência venha aumentando globalmente (SILVA DR, et al, 2018).

Os resultados mostraram maior prevalência de TB da forma pulmonar (51\%), o que corrobora os dados da literatura (ARNEDO-PENA A, et al., 2019; SUNNETCIOGLU A, et al. 2015). A prevalência da distribuição da TB extrapulmonar diferiu de outros estudos com relação à distribuição dos órgãos e sistemas acometidos (SUNNETCIOGLU A, et al. 2015; AYED HB, et al., 2019; VARGHESE B E AL-HAJOJ S, 2015), devendo ser avaliada regionalmente, para que as estratégias terapêuticas possam ser melhor planejadas, considerando as especificidades do tratamento de cada tipo de TB extrapulmonar (BRASIL, 2018).

A TB-MDR continua sendo uma ameaça à saúde pública (WORLD HEALTH ORGANIZATION, 2019), constituindo um problema emergente em todo o mundo, com incidência cada vez maior (LAPAUSA MR, 2013). Seu tratamento deve ser adaptado às necessidades individuais, pois os diferentes padrões de resistência exigem abordagens específicas. O acompanhamento dos indivíduos com TB-MDR deve ser realizado nos centros de referência (BRASIL, 2018).

Um estudo realizado no CRT-TB do ES, no período de 2002 a 2012, mostrou prevalência de $5 \%$ de TBMDR (FREGONA GC, et al., 2017). No presente estudo, realizado no mesmo CRT-TB, houve prevalência de $10,2 \%$, o que mostra aumento ao longo desse período. Este resultado é preocupante, mas corrobora com os dados mundiais de aumento da prevalência da TB-MDR nas últimas décadas (LAPAUSA MR, 2013). 
Os resultados mostraram que $100 \%$ dos indivíduos com TB-MDR apresentaram baciloscopia positiva. Além disso, foi encontrada associação entre a baciloscopia e a classificação da TB com relação à resistência às drogas, mostrando que o grupo TB-MDR foi mais propenso a apresentar baciloscopia positiva do que 0 grupo que não apresentava resistência às drogas. Esse resultado corrobora dados da literatura, que têm demonstrado que os indivíduos TB-MDR apresentam baciloscopia positiva com maior frequência (FREGONA GC, et al., 2017). Esses dados merecem atenção, e devem ser considerados na organização dos fluxos de atendimento, considerando que os indivíduos bacilíferos têm maior potencial de transmissibilidade da doença, perpetuando a cadeia de transmissão justamente da TB-MDR, que apresenta maior complexidade de tratamento (BRASIL, 2018), constituindo um dos maiores desafios mundiais para o enfrentamento da TB (SILVA DR, et al., 2018).

A baciloscopia do escarro, desde que executada corretamente, tem sensibilidade de $50 \%$ e especificidade de $80 \%$ (MOLICOTI P, et al., 2014), o que tem grande importância epidemiológica, pois os casos com baciloscopia positiva são os maiores responsáveis pela sustentação da cadeia de transmissão (BRASIL, 2018). Neste estudo, foi observada associação entre a baciloscopia e a cultura. No grupo de indivíduos com baciloscopia positiva, houve um maior percentual de cultura positiva do que no grupo com baciloscopia negativa, o que era esperado. Apesar disso, $31,8 \%$ dos indivíduos apresentaram baciloscopia negativa, com cultura positiva. Esse resultado é compreensível, considerando-se que a cultura do escarro pode aumentar em até 30\% o diagnóstico bacteriológico da doença (BRASIL, 2018), pois apresenta maior sensibilidade $(84,9 \%)$ e especificidade $(99,5 \%)$, sendo considerada padrão-ouro para o diagnóstico da TB (MOLICOTI P, et al., 2014).

A radiografia do tórax em indivíduos com diagnóstico bacteriológico tem como objetivos excluir outra doença pulmonar associada, avaliar a extensão do acometimento e sua evolução radiológica durante o tratamento (BRASIL, 2018). Todos os indivíduos com diagnóstico de TB pulmonar, associada ou não à extrapulmonar, apresentaram diagnóstico suspeito na radiografia de tórax, enquanto nos indivíduos com a forma extrapulmonar isolada, foi observada radiografia suspeita em $41,2 \%$ dos casos. Dados semelhantes foram descritos em outro estudo, que apresentou prevalência de $99,3 \%$ de radiografia de tórax suspeita na forma clínica pulmonar e 47,5\% na forma clínica extrapulmonar (ARNEDO-PENA A, et al., 2019).

As principais limitações do estudo foram o pequeno número amostral e o período de apenas seis meses de coleta de dados, além disso, por tratar-se de um estudo com coleta de dados secundários, obtidos nos prontuários, o risco de viés é aumentado devido à imprecisão e ausência de algumas informações.

\section{CONCLUSÃO}

Neste CRT-TB, houve maior prevalência de indivíduos do sexo masculino, adultos, de raça parda, residentes na região Metropolitana, com a forma pulmonar da TB. Entre as formas extrapulmonares houve maior prevalência da ganglionar periférica. A comorbidade mais prevalente foi o tabagismo, seguida de etilismo, HAS, doença hepática, DM, HIV e drogas ilícitas. A baciloscopia positiva apresentou associação com a cultura positiva e com a TB-MDR. Todos os indivíduos com TB-MDR apresentaram baciloscopia positiva, o que merece atenção, considerando que os indivíduos bacilíferos possuem maior potencial de transmissibilidade da doença. Não foram encontradas associações do resultado da baciloscopia com outras variáveis. A TB da forma pulmonar apresentou maior prevalência de radiografia de tórax suspeita, e dentre os indivíduos extrapulmonares, apresentaram maior prevalência de radiografia de tórax suspeita os tipos Pleural/Pericárdica.

\section{REFERÊNCIAS}

1. ARNEDO-PENA A, et al. Pulmonary Versus Extrapulmonary Tuberculosis Associated Factors: A Case-Case Study. Microbiology insights. 2019; 12: 1-10.

2. AYED HB, et al. Epidemiology and disease burden of tuberculosis in south of Tunisia over a 22-year period: Current trends and future projections. PloS One. 2019; 14 (7): e0212853.

3. BRASIL. Ministério da Saúde. Secretaria de Vigilância em saúde. Bol Epidemiol. 09. $2019 ; 50$ (mar.2019):1-18. Disponível em: https://www.saude.gov.br/images/pdf/2019/marco/22/2019-009.pdf. 
4. BRASIL. Ministério da Saúde. Guidelines for tuberculosis control in Brazil. 2018. 2. Disponível em: http://bvsms.saude.gov.br/bvs/publicacoes/manual_recomendacoes_controle_tuberculose_brasil_2_ed.pdf

5. BRASIL. Ministério da Saúde (BR). Secretaria de Vigilância em saúde. Bol Epidemiol, $201 \overline{6} ; 47$ (13): 1-15.

6. BRASIL. MS/SVS. Sistema de Informação de Agravos de Notificação. [base de dados online]. Sinan Net (dataSUS). 2018 [acesso em 27 novembro 2018]. Disponível em: tabnet.datasus.gov.br

7. CARAFFA E, et al. The diabetes-tuberculosis co-epidemic: the role of international migration. The International Journal of Tuberculosis and Lung Disease, 2016; 20 (6): 771-777.

8. CHUNG W-S, et al. Tuberculosis increases the subsequent risk of acute coronary syndrome: a nationwide populationbased cohort study. The International Journal of Tuberculosis and Lung Disease, 2014; 18 (1): 79-83.

9. CRABTREE-RAMÍREZ B, et al. HIV-related tuberculosis: mortality risk in persons without vs. with culture-confirmed disease. The Inter J Tuberc Lung Dis, 2019; 23 (3): 306-314.

10. CRUZ, VD, et al. Crack consumption and tuberculosis: an integrative review. SMAD. Revista eletrônica saúde mental álcool e drogas, 2013; 9 (1): 48-55.

11. FERNANDES FMC, et al. Relationship between climatic factors and air quality with tuberculosis in the Federal District, Brazil, 2003-2012. Brazilian Journal of Infectious Diseases, 2017; 21 (4): 369-375.

12. FREGONA GC, et al. Fatores associados à tuberculose resistente no Espírito Santo, Brasil. Rev de Saúde Pública, 2017; 51 (41): 1-11.

13. HADDAD L, et al. M. Characteristics of Liver Transplantation in Argentina: A Multicenter Study.Transplantation proceedings. 2018; 50 (2): 478-484.

14. IMTIAZ S, et al. Alcohol consumption as a risk factor for tuberculosis: meta-analyses and burden of disease. Eur Respir J, 2017; 50 (1): 1700216.

15. JEON CY, MURRAY MB, et al. Diabetes Mellitus Increases the Risk of Active Tuberculosis: A Systematic Review of 13 Observational Studies. Medicina PLoS, 2008; 5 (7): 1091-1101.

16. KHAN AH, et al. Smoking on treatment outcomes among tuberculosis patients. The American Journal of the Medical sciences, 2015; 349 (6): 505-509.

17. KUMARI P, MEENA LS. Factors affecting susceptibility to Mycobacterium tuberculosis: a close view of immunological defence mechanism. Applied biochemistry and biotechnology, 2014; 174 (8): 2663-2673.

18. LAPAUSA MR, et al. Nuevos fármacos antituberculosos en la tuberculosis resistente y multirresistente. Medicina Clínica, 2013; 141 (7): 306-313.

19. LEONG LY, et al. Risk factors of tuberculosis after liver transplant in a tertiary care hospital. Journal of Microbiology, Immunology and Infection, 2019; in press.

20. LEUNG CC, et al. Diabetic control and risk of tuberculosis: a cohort study. American Journal of Epidemiology, 2008; 167 (12): 1486-1494.

21. MOLICOTI P, et al. Custo-efetividade no diagnóstico da tuberculose: escolhas em países em desenvolvimento. $O$ Jornal de Infecção nos Países em Desenvolvimento. 2014; 8 (1): 024-038.

22. OLIVA, HN, et al. Estudo epidemiológico da tuberculose no estado de Minas Gerais. Revista Eletrônica Acervo Saúde, 2019; (18), e78-e78.

23. PRÉCOMA DB, et al. Atualização da Diretriz de Prevenção Cardiovascular da Sociedade Brasileira de Cardiologia 2019. Arq Bras Cardiol. 2019; 113 (4): 787-891.

24. ROCHA NP, et al. Diabetes mellitus em pacientes com tuberculose internados em hospital de referência em Belo Horizonte, Minas Gerais. Rev Med Minas Gerais, 2016; 26 (5); S65-S71.

25. SEEGERT AB, et al. Tuberculosis and hypertension: a systematic review of the literature. International journal of infectious diseases, 2017; 56: 54-61.

26. SILVA DR, et al. Risk factors for tuberculosis: diabetes, smoking, alcohol use, and the use of other drugs. J Bras de Pneumol, 2018; 44 (2): 145-152

27. SUNNETCIOGLU A, et al. Comparative analysis of pulmonary and extrapulmonary tuberculosis of 411 cases. Annals of clinical microbiology and antimicrobials, 2015; 14 (1): 34.

28. UGARTE-GIL C, et al. Diabetes mellitus among pulmonary tuberculosis patients from four TB-endemic countries: the TANDEM study. Clinical Infectious Diseases, 2020; 70 (5): 780-788.

29. VARGHESE B, AL-HAJOJ S. Mapeamento da epidemiologia e tendências da tuberculose extra-pulmonar na Arábia Saudita. Revista internacional de micobacteriologia, 2015; 4 (4): 261-269.

30. WORLD HEALTH ORGANIZATION. Global tuberculosis report, 2019. WHO/CDS/TB/2019.15. Geneva, Switzerland: WHO, 2019.

31. WORLD HEALTH ORGANIZATION. Moscow declaration to end TB. 2017; 16-17. 